\title{
La dynamique expérientielle et identitaire des enseignants associés en enseignement professionnel
}

\section{Problématique}

Le présent article résume une recherche doctorale menée auprès de formateurs d'enseignants, soit des enseignants associés (EA), du secteur de l'enseignement professionnel au Québec. Cette étude découle de constatations indiquant que la transition qu'effectuent les enseignants de la formation professionnelle du métier vers l'enseignement entraine des transformations importantes en matière de compétences professionnelles, d'identité professionnelle, d'environnement de travail et d'interactions (Balleux, 2013; Deschenaux et Roussel, 2008; Gagné, 2015; Gaudreault, 2011). Les nouveaux espaces fréquentés, avec les situations vécues et les interactions établies, influencent la façon dont l'individu se perçoit professionnellement et sa manière d'agir (Perez-Roux, 2011). Dans le continuum des parcours professionnels incluant les formations suivies, ces recherches ne prennent cependant pas en considération le rôle d'EA. Pourtant, plusieurs enseignants s'étant insérés dans la profession au point d'en arriver à une forme d'adaptation et de régulation de leur enseignement (Balleux et Gagnon, 2011) choisissent de former la relève. Ces EA, anciens travailleurs de métier et toujours enseignants en formation professionnelle, doivent alors s'approprier un nouveau rôle professionnel, celui de formateur d'enseignants (Portelance, Gervais, Lessard et Beaulieu, 2008). Ils s'engagent ainsi dans de nouvelles transformations sur les plans expérientiel et identitaire. Dans le contexte où la formation continue destinée aux EA n'est ni formalisée pour l'ensemble des universités ni systématiquement adaptée au contexte de l'enseignement professionnel (Deschenaux, Monette et Tardif, 2012), elle fournit donc un encadrement limité. Par conséquent, quelle interprétation les EA 
font-ils de leur rôle de formateur? Quels évènements significatifs de leur vécu professionnel contribuent à cette interprétation? Ces questions mettent en lumière le peu d'informations dont disposent les milieux professionnels et universitaires à propos de ces formateurs qui participent significativement à la formation de la relève enseignante (Deschenaux et al., 2012).

\section{Cadre de référence}

Répondre aux interrogations soulevées exige de mobiliser divers fondements théoriques. D'une part, la théorie des identités (Burke et Stets, 2009) permet d'appréhender l'interprétation que les EA font de leur rôle au regard de leurs multiples identités, celles de métier, d'enseignant et de formateur d'enseignants. La recherche se distingue ainsi des travaux sur la transition vers l'enseignement qui ne prennent en compte qu'une ou deux identités professionnelles. D'autre part, la notion d'expérience (Zeitler et Barbier, 2012) contribue à circonscrire l'apport du vécu professionnel de l'EA depuis le métier jusqu'à la formation des stagiaires. L'attention se porte alors sur la forme discursive de l'expérience qui permet de relater et de saisir les évènements et les interactions qui ont marqué leurs parcours professionnels.

\section{Cadre méthodologique}

Sur le plan méthodologique, cette recherche interprétative s'appuie sur une perspective biographique cohérente à l'étude des parcours professionnels (Demazière, 2011). Elle fait appel à différentes stratégies de collecte et d'analyse pour assurer la validité de la recherche et enrichir théoriquement et empiriquement le phénomène de la dynamique expérientielle et identitaire des EA. Un questionnaire électronique a été rempli par $186 \mathrm{EA}$ de partout au Québec (Fenneteau, 2015). Ce questionnaire, en plus de dresser le portrait de ce groupe, a permis de recruter 16 EA volontaires pour réaliser deux phases d'entretiens biographiques (Demazière, 2011). Au cours de la première phase, la chercheure et les participants ont reconstruit le parcours professionnel de ces derniers, tout en y identifiant des évènements déterminants. Au cours de la seconde phase, les évènements ciblés ont été approfondis pour déterminer leur contribution par rapport à l'interprétation du rôle d'EA. Une analyse de contenu a été réalisée pour le questionnaire, alors qu'une démarche d'analyse structurale a été effectuée sur les verbatims des entretiens.

\section{Résultats}

Les résultats présentés constituent un aperçu des principaux constats dégagés au cours de la recherche doctorale. À propos de l'interprétation de leur rôle, près de la moitié des EA affirment ne pas se percevoir comme des formateurs d'enseignants. Ils déclarent plutôt: "Je me perçois plus comme un accompagnateur qu'un formateur. La plupart de mes stagiaires sont déjà enseignants. Ils ont des connaissances, de l'expérience et des compétences» (EA70). Puisque les EA et les stagiaires du secteur professionnel enseignent généralement tous les deux, il semble particulièrement difficile pour les EA de se construire une identité de formateur d'enseignants. 
Concernant les évènements et les interactions significatifs de leur vécu professionnel, les EA évoquent l'importance du soutien reçu de la part d'un collègue lors de leur propre insertion dans la profession. Un EA mentionne : «Il [le collègue] m’a dit de tenir un bon cinq ans. Ça m'a aidée qu’il méencourage à tenir un an ou deux de plus et regarde où cela m'a menée » (EA1). Les participants réfèrent également au soutien offert par les EA les ayant accompagnés pendant leurs stages de formation. Comme le montre l'extrait, l'aspect du soutien moral prime sur celui de la formation, ce qui souligne la complexité du rôle d'EA et rejoint la difficulté à se définir en tant que formateur d'enseignants. Plus encore, la formation continue en accompagnement et les collègues EA ne sont pas apparus comme particulièrement marquants.

\section{Conclusions}

La recherche conclut à une interprétation du rôle d'EA éminemment individuelle, car cette interprétation s'enracine principalement dans des évènements et des interactions antérieurs aux fonctions d'EA. Les EA en exercice ont également peu l'occasion de confronter l'interprétation qu'ils font de leur rôle avec leurs homologues. De ce fait, l'identité de l'EA, qui oscille entre formateur et accompagnateur, s'en voit fragilisée. Les recommandations avancées par la recherche doctorale proposent donc de réfléchir à la conjugaison entre les facettes d'accompagnement et de formation inhérentes au rôle d'EA. Elles suggèrent de développer une vision partagée de ce rôle au sein des instances universitaires et des milieux de pratique afin notamment de favoriser l'arrimage entre le mandat confié aux EA et la réalité de leur contexte d'exercice auprès des stagiaires.

\section{Références}

Balleux, A. (2013). La transition entre le métier et l'enseignement des nouveaux enseignants de formation professionnelle au Québec : un passage à comprendre et à accompagner (Rapport scientifique intégral). Repéré à http://www.frqsc.gouv. qc.ca/documents/11326/449044/PT BalleuxA rapport+2013 transition+m\%C3\%A9tier+enseignant/cd4039e2$\underline{8 d 5 f-411 e-9 d 8 b-a d 4 b 064 a d e 5 d}$

Balleux, A. et Gagnon, C. (2011). L'accompagnement dans la transition entre l'exercice d'un métier et son enseignement. Dans F. Lacourse, S. Martineau et T. Nault (dir.), Profession enseignante : démarches et soutien à l'insertion professionnelle (p. 153-166). Anjou, QC : CEC.

Burke, P. J. et Stets, J. E. (2009). Identity theory. New York, NY : Oxford University Press.

Demazière, D. (2011). L'entretien biographique et la saisie des interactions avec autrui. Recherches qualitatives, 30(1), 61-83. Repéré à https://hal.archives-ouvertes.fr/hal-01510177

Deschenaux, F., Monette, M. et Tardif, M. (2012). État de la situation de la formation à l'enseignement professionnel au Québec (Rapport final). Repéré à http://www.treaqfp.qc.ca/103/PDF/Rapport final Groupe reflexion FP janv12.pdf

Deschenaux, F. et Roussel, C. (2008). L'accès à la carrière enseignante en formation professionnelle au secondaire : le choix d'un espace professionnel. Nouveaux cahiers de la recherche en éducation, 11(1), 1-16. http://dx.doi.org/10.7202/1017506ar

Fenneteau, H. (2015). Enquête : entretien et questionnaire (3éd.). Paris : Dunod.

Gagné, A. (2015). Les valeurs issues des carrières initiales des enseignants en formation professionnelle : leurs rôles dans le développement d'une nouvelle identité professionnelle d'enseignant (Mémoire de maitrise). Accessible par ProQuest Dissertations \& Theses. (10010912) 
Gaudreault, A. (2011). L'identité professionnelle des enseignants en formation professionnelle ayant débuté à enseigner après plusieurs années d'expérience dans un métier : portrait d'un processus (Mémoire de maitrise, Université du Québec à Chicoutimi). Repéré à https://constellation.uqac.ca/240/1/030165959.pdf

Perez-Roux, T. (2011). Changer de métier pour devenir enseignant : transitions professionnelles et dynamiques identitaires. Recherches en éducation, (11), 39-54. Repéré à https://hal.archives-ouvertes.fr/hal-01715841

Portelance, L., Gervais, C., Lessard, M. et Beaulieu, P. (2008). La formation des enseignants associés et des superviseurs universitaires - Cadre de référence (Rapport de recherche). Repéré à https://www.usherbrooke.ca/education/fileadmin/ sites/education/documents/Intranet/Documents officiels/Rapport Cadre reference 2009.pdf

Zeitler, A. et Barbier, J.-M. (2012). La notion d'expérience, entre langage savant et langage ordinaire. Recherche et formation, (70), 107-118. http://dx.doi.org/10.4000/rechercheformation.1885

\section{Pour citer cet article}

Gagné, A. (2019). La dynamique expérientielle et identitaire des enseignants associés en enseignement. Formation et profession, 27(1), 124-127. http://dx.doi.org/10.18162/fp.2019.a164 